\title{
Techno-Economic Analysis of a Software-Defined Optical Media Contribution and Distribution Network
}

\author{
Bram Naudts*, Marlies Van der Wee*, Luc Andries ${ }^{\dagger}$, Sofie Verbrugge*, Didier Colle*, \\ ${ }^{*}$ Dept. of Information Technology (INTEC) \\ Ghent University - iMinds, Ghent, Belgium \\ Email: \{bram.naudts, marlies.vanderwee, sofie.verbrugge, didier.colle $\} @$ intec.ugent.be \\ ${ }^{\dagger}$ SDNsquare \\ Ghent, Belgium \\ Email: luc.andries@sdnsquare.com
}

\begin{abstract}
Bringing video images from camera to screen is a complex production process involving several intermediate steps and actors. The media sector is characterized by a high need for fast and reliable data transport, between production locations, post-production facilities, broadcasters and back-up locations. The growing size of video files and the stringent network requirements for live production have given rise to a combination of transport solutions including physical transport of raw materials, transport of proxies via best-effort Internet Protocol (IP) networks and transport of live video streams via Serial Digital Interface (SDI) over microwave, satellite, leased lines or optical fiber. Due to this fast-paced evolution, media companies face high investments costs for upgrading both end devices (e.g. cameras) and network transport infrastructure. As such, the cost of maintaining and upgrading a multitude of transport networks can be prohibitive.

The MECaNO project [1] proposes a lossless packet switching technology developed that can guarantee delivery deadlines in order to create a single, lossless, IP-based production system which is realized by leveraging technological advances such as the decreasing cost of optical technology and the flow-based softwaredefined networking (SDN) architecture. Apart from explaining the MECaNO solution, the goal of this paper is to quantify the economic impact of the proposed technological solution. The results show that the cost for the optical infrastructure (L1) remains relatively stable while the cost for $\mathrm{L} 2-\mathrm{L3}$ technologies drops significantly leading to a total cost reduction of $27 \%$.
\end{abstract}

\section{INTRODUCTION TO THE MEDIA ACTOR LANDSCAPE}

Media production is a complex process. The following steps can be recognized in the content creation process (Figure 1, based on [2]): (1) development of the concept, budgeting, financing and scheduling; (2) pre-production which includes all the practical aspects before the actual shooting such as scripting, discussion with crew members and talents, etc.; (3) production: the actual shooting in the studio or on location, the created materials (rushes) are on a daily basis (dailies) turned into lower resolution materials (proxies) which are used by the director for selection and quality assurance purposes; (4) post-production, containing to make the footage ready for final delivery such as editing, sound design, color grading, special effects, (sub)titling; (5) distribution to the audience (playout, video-on-demand, web, social media) and (6) archiving. From the very beginning to the end, (7) the sales and marketing business process takes place. For marketing purposes, stills and trailers have to be produced. The producer is also involved to negotiate a distribution agreement, and as such needs access to all content on request. The media production process in its entirety involves a variety of actors (production houses, facility providers, broadcasters, technology support providers, content providers, etc.) that need to collaborate and exchange data throughout the process. The media creation process used to rely on a system where tapes were transferred manually from the place of creation to the place of editing and finally to the playout system. As this process has become highly collaborative in nature, being able to use file-based workflows has opened up more creative and efficient workflows. The authors of [3] for example, describe a IP-based production network with quality of service provisioning through traffic engineering. Today, several content production areas already take advantage of the availability of higher network bandwidths by setting up file-based systems and fully networked editing environments. Sohonet, for example, connects multiple mediaoriented companies in a specific location in London. Sohonet has started 15 years ago as a research project that involved several London based post production facilities [4]. Now, Sohonet is an independent media network having a global reach with offices in the UK, Los Angeles and Sydney. They give their media customers the opportunity to use tapeless production by offering high bandwidths, data security and expertise in workflows and techniques. Technological enablers of this shift are the decreasing cost of course/dense wavelength division multiplexing (CWDM/DWDM)-based optical networks and the availability of IP-based production systems. An essential exception to this shift are live production systems [5]. Due to the need for seamless transport of live content, a best-effort network approach as delivered by IP-based production systems is insufficient.

As a result, technologies such as SDI and coaxial cable that can guarantee constant bandwidth, high availability 


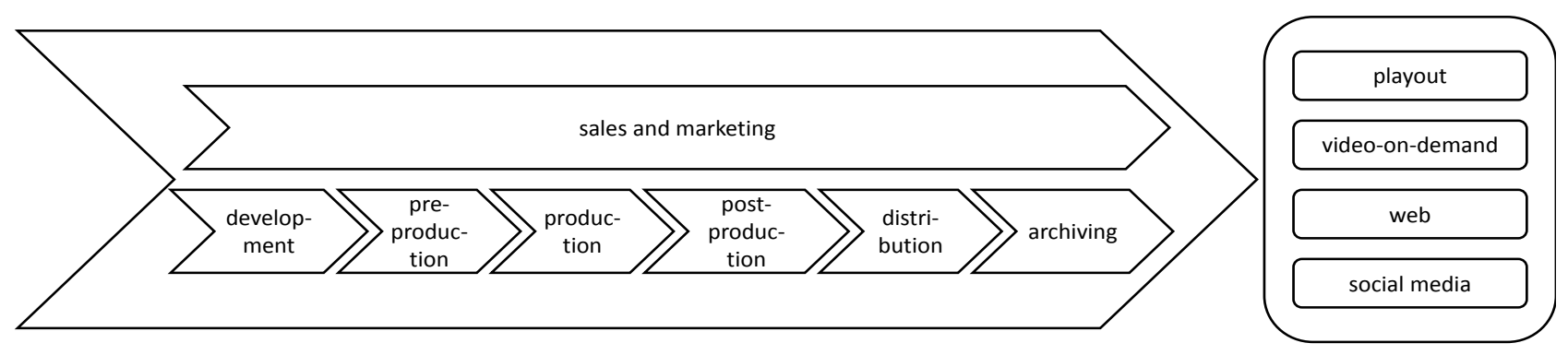

Fig. 1. Overview of the media creation process flow

and low latency are still used for live video transmission. However, with the growing demand for higher frame rates, higher dynamic range and higher resolutions, the additional investment in hardware and cabling for conventional live video transmission implementations becomes a financial hurdle for content producers. Therefore, in the MECaNO project, a lossless packet switching technology is proposed with as goal to create an IP-based production system which can be used for both non-live and live content. As part of this project, a technoeconomic evaluation [6] has been conducted to estimate the cost advantage for content providers as they migrate from high definition (HD) video production towards $4 \mathrm{~K}$ video production.

The remainder of the paper is structured as followed. First, an overview is given of current media contribution and distribution networks. Second, we detail the main challenges to realize a converged media production system and describe the proposed alternative which is based on the availability of a dedicated CWDM/DWDM optical network and SDNbased flow control. Third, we detail the techno-economic study before we finally conclude the article.

\section{Media CONTRIBUtion AND DistribUtion NETWORKS TODAY}

Professional media production uses a diverse set of transport options, each option with its own right of existence.

The transport of tapes and disks can be done by physical transport of the media bearer. The transport is done by ship, car or moped. The amount of data transferred is nearly unlimited with this transport option. It is very efficient for large amounts of data over short distances (not live). A second option is the use of best-effort Internet connections. As these typically have a low bandwidth (10-100 megabits per second, Mbps), they are used to transmit low resolution proxies of the rushes (e.g. Standard Definition, SD). This is often done via non-accelerated file transfer solutions such as WeTransfer [7]. A third option, often used for live reporting on location during events, are microwave transmissions. These use a radio connection between two antennas to transfer data. The antennas require line of sight. The maximal reach of this type of connection is $75 \mathrm{~km}$ but in practice, this is around
$30 \mathrm{~km}$. It is possible to set up a temporary radio transmission between two points in a relatively short time (half a day). Microwave transmissions can provide a guaranteed line rate of 2x100 Mbps. The fully redundant 100Mbps connection can be allocated as needed. Mobile transmission requires a specialized Outside Broadcasting van (OB van). An alternative to microwave transmissions are satellite transmissions, used to transmit a signal from any recording location. Because of their fast setup time, they are mainly used for news gathering. Typical channel slots are $9 \mathrm{MHz}$ for $\mathrm{SD}$ and $18 \mathrm{MHz}$ for $\mathrm{HD}$ media. Satellite transmissions also require a specialized van (OB van with satellite transponder) and have a line rate of 7Mbps. Finally, to interconnect static locations, managed leased lines can be used (e.g. IP/MPLS) or a leased dark fiber. To increase performance of these lines, proprietary accelerated file transfer solutions such as Aspera, Signiant and FileCatalyst are used, relying on very aggressive, UDP-based protocols instead of regular TCP-based protocols. These bursty protocols aim at filling the network independent of the infrastructure, any other traffic and distance, which makes them useful for transferring the data as fast as possible.

Today, to transport live video, legacy video networks are used such as SDI and coaxial cable. The current legacy video networks are designed as fit-for-purpose solutions. As such, each link typically only contains a single video stream and preserves timing information. There is no real need for buffering as the transport network is dimensioned to cope with the line rate of both input (e.g. camera) and output (e.g. a post-production facility) devices. As a result, there is no interference, oversubscription or data loss. The deployment, operations, maintenance and migration cost of hardware and cabling for this multitude of networks is however significant, and can even be prohibitive. More and more media companies are therefore looking into converting their video networks to an IP-based network. There however are still a lot of challenges and there is no plug-and-play technology that can provide the same functionality today. The simplest solution is to replace the SDI cable for a single video stream with an Ethernet cable also carrying just a single video stream. However, this brings no real benefit as IP networks provide a best effort service without any service guarantees. The goal thus is to 


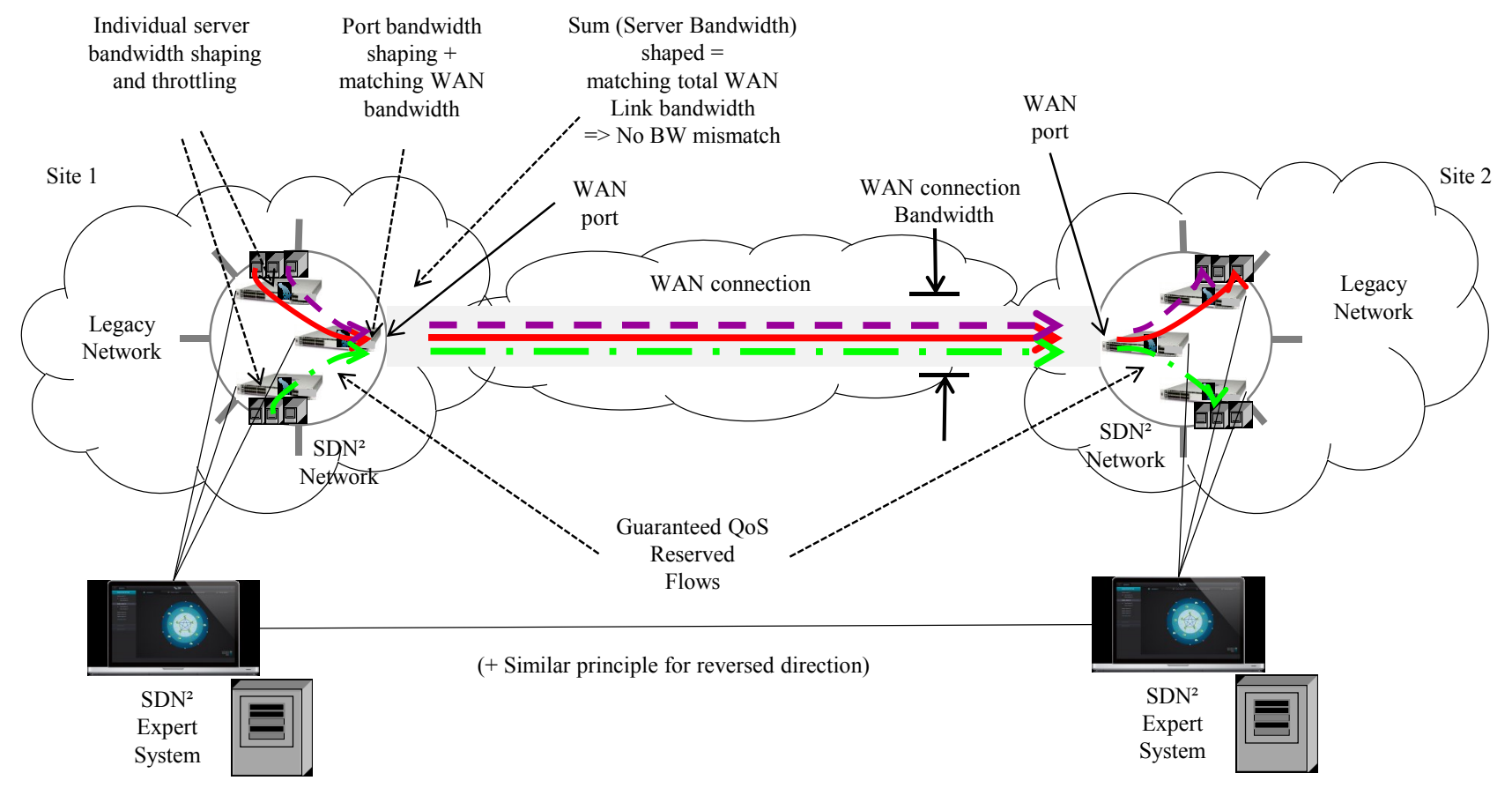

Fig. 2. Overview of the mechanism to reach a SDN-based optical media contribution and distribution network

build an IP network with the flexibility of IP networks but with the service guarantees of a legacy video network (e.g. an SDI network). This means an IP network that is able to provide lossless packet switching for huge media files (in the order of multiple Gbps) while being capable of delivering just-in-time workflows and guaranteed performance in terms of packet loss, timing (e.g. impossible for live events to buffer), synchronization (e.g. of video and audio streaming) and latency without increasing costs nor network complexity.

\section{A CONVERGED, IP-BASED MEDIA CONTRIBUTION AND DISTRIBUTION NETWORK}

Understanding how a converged IP-based media production system that can be used for both live and non-live production requires an insight in the components of the end-to-end IP network path. In essence, when transport of a media file occurs between two locations, traffic will originate in one local area network (LAN) in which it is captured (e.g. a micro, a camera) and go through a (potentially high) number of switches, routers and other middle boxes (wide area network, WAN) to the LAN network of the second location where the data stream is displayed, edited, stored, etc. In such a network design, the limiting factor in terms of bandwidth is typically the WAN link. As operators usually apply statistical multiplexing and perform intermediate packet processing steps it remains impossible to offer performance guarantees when using an operator managed WAN link. Therefore, the proposed solution requires a connection with guaranteed bandwidth such as (a concatenation of) unmanaged leased lines or dark fiber connec- tions ${ }^{1}$. As such a hard upper bound for the available throughput in the end-to-end path is the bandwidth of the ingress WAN port $^{2}$ and action should be undertaken to avoid oversubscription on this line. However, even with the WAN network under control, mismatches in terms of bandwidth demand are still likely to occur between either of the LAN networks and the WAN network. Several sources from one LAN network may be sending traffic to the same destination leading to a competition for bandwidth in the destination network resulting in packet drops and when needed retransmissions making the transport of live media over such a network impossible. As such, the SDN technology as used in the MECaNO project, uses a combination of counter measures at both the ingress and egress points of the network. First, to overcome possible mismatches, a network management system is used to reserve, in advance, bandwidth between two (or possibly more) locations. This reservation system should avoid oversubscription. However, even when a bandwidth is reserved or provisioned on the WAN link for a certain media transfer flow, the actual source of the media flow is not aware of this reservation. In most cases, the physical bandwidth of the source port, connected to the LAN source network has no relation and doesnt match the bandwidth provisioned on the WAN link. Hence the source pumps data traffic in the source network at its own local

\footnotetext{
${ }^{1}$ In case of dark fiber the available bandwidth depends on many factors including the chosen technology (e.g. CWDM or DWDM), the distance between both locations, etc. Guaranteed bandwidth therefore refers to the attainable bandwidth over a single fiber channel (e.g. 1Gbps or 10Gbps).

${ }^{2} \mathrm{We}$ assume an unidirectional link
} 
full bandwidth speed. When this traffic arrives at the ingress WAN port, a possible speed mismatch occurs between the source port and the bandwidth provisioned at the WAN port. This normally results in packet-loss, with the above described impact on the utilization efficiency of the WAN link. On top of this, bandwidth mismatched could already occur in the source LAN itself, before reaching the ingress WAN port, leading to the same effect. Therefore, the second countermeasure of the SDN technology is to limit the traffic of the source to the bandwidth provisioned on the WAN link for this media flow (or any bandwidth bottleneck in the source LAN network if that bandwidth would be lower than the WAN bandwidth). In case multiple sources are sending traffic to the same WAN port, the SDN technology will limit each individual flow in such a way that the total sum of the source bandwidths fits the provisioned WAN bandwidth. Third, in order to allow for differentiated service guarantees, the technology uses multiple independent queues (e.g. within a single optical DWDM channel/line). Fourth, per queue, a flow class can be defined to which priorities can be assigned. By doing so, the network can guarantee strict performance guarantees for live traffic while at the same time best effort traffic can pass through the same channel/link. Fifth, by smoothing traffic, packet loss due to micro burst is eliminated. Finally, in order to be able to provide service guarantees, the proposed functionality needs to be deployed at each location which requires specific QoS capable switches in the LAN networks.

\section{CAlculating The COST SAVINGS OF NETWORK CONVERGENCE}

Taking into account the technological description of the legacy system in section II and of the proposed solution in the MECaNO solution in section III, this section details a technoeconomic study aimed at calculating the infrastructure cost savings in a media network that are realizable in a wide area network (WAN) via:

1) network convergence: by migrating the plethora of single-purpose networks (in this case an SDI network and an IP network) to a single, high-bandwidth, multipurpose IP-based transmission network with performance guarantees

2) decoupling the network from the production format: by decoupling the infrastructure from the media production devices, the infrastructure becomes format agnostic and will be able to support future resolutions and services

\section{A. Scenarios}

In each of the scenarios, a leased dark fiber connection is interconnecting the different hubs. A DWDM-based optical cross connect $(\mathrm{OXC})$ is used to route the optical signals. On top of the optical infrastructure a different set of technologies is used to route the traffic sources to their destination in each of the following two scenarios:

1) As a starting point, the situation today is used with two different parallel networks supporting HD streams, an
SDI network with strict performance guarantees for live production and an IP network with without performance guarantees (best effort) that is used for media without stringent time constraints. In scenario 1 , the network is upgraded to be $4 \mathrm{~K}$ compatible. This is modelled by scaling the original traffic sources with a factor of 4 in respect to the situation today and adding additional equipment or replacing equipment as needed. IP/MPLS cards are used to realize the QoS IP network and SDI technology for the live media production network.

2) The second scenario has the same starting point in terms of traffic sources. However, the original SDI streams (associated with live traffic) are now routed over the IP network. This is made possible by using the MECaNO technology (see section III). Similarly to the first scenario, traffic sources are scaled with a factor of 4 to reflect the shift from HD towards $4 \mathrm{~K}$ media production.

\section{B. Network design}

In order to make a realistic estimation of network costs and typical traffic flows, we interconnect three locations in Flanders that are also in real productions interconnected for media transport: the offices of Radio 2 in Ghent, the central production and storage hub of VRT in Brussels and the postproduction facilities of Videohouse in Vilvoorde. Both the Ghent and Vilvoorde location are directly connecte to the Brussels location. The focus of the cost study is on the node located in Ghent. We assume a bit rate of 10 gigabit per second (Gbps) per DWDM channel is attainable on the dark fiber link.

\section{Traffic sources}

The considered traffic flows and background traffic flows that need to be transported over these links are summarized in Table I.

TABLE I

OVERVIEW OF TRAFFIC FLOWS

\begin{tabular}{|c|c|c|}
\hline Description & \multicolumn{2}{|c|}{ bandwidth (Mbps) } \\
\hline \multicolumn{3}{|c|}{ Ghent-Brussel-Vilvoorde } \\
\hline Proxies & 100 & 400 \\
\hline Rushes & 500 & 2000 \\
\hline Aspera-generated traffic & 1000 & 0 \\
\hline 3 SDI flows (compressed) & 400 & 1600 \\
\hline \multicolumn{3}{|l|}{$\begin{array}{l}\text { Vilvoorde-Brussel-Ghent } \\
\end{array}$} \\
\hline Aspera rushes & 1000 & 0 \\
\hline Aspera-generated traffic & 1000 & 0 \\
\hline 3 HD SDI flows (compressed) & 400 & 1600 \\
\hline \multicolumn{3}{|l|}{ Vilvoorde-Brussels } \\
\hline Edge rushes (from VRT storage) & 1485 & 6000 \\
\hline Edge proxies & 200 & 800 \\
\hline Remote editing & 1485 & 6000 \\
\hline \multicolumn{3}{|l|}{ Background traffic } \\
\hline 18 SDI streams to Brussels or Vilvoorde & 1485 & 5940 \\
\hline 18 SDI streams from Brussels or Vilvoorde & 1485 & 5940 \\
\hline 4 IP streams to Brussels or Vilvoorde & 100 & 400 \\
\hline 4 IP streams from Brussels or Vilvoorde & 100 & 400 \\
\hline 4 IP streams to Brussels or Vilvoorde & 1000 & 4000 \\
\hline 4 IP streams from Brussels or Vilvoorde & 1000 & 4000 \\
\hline
\end{tabular}




\section{Cost input data}

Cost values are often a point of discussion in technoeconomic studies. To tackle this point we obtained cost values from official price lists of several vendors and validated these with industry experts. The used cost model for a node is separated in an optical part (DWDM-based optical node) and an electronical part (SDI or IP-based node) that uses the optical network. We estimate the cost of one DWDM-based optical node with 40 wavelengths at $€ 32,500$ or a cost per optical wavelength of $€ 813$. We estimate the total cost for 48 (8x6) uni-directional HD SDI channel at $€ 111,500$ or a cost per uni-directional HD SDI channel of $€ 2,322$. 4K SDI is fourtimes the data rate of HD SDI and has not been standardized so far. We assume that 4 parallel HD SDI channels are used to realize $4 \mathrm{~K}$ SDI and estimate the cost for $4 \mathrm{~K} \mathrm{SDI}$ at 4 times the cost of HD SDI $(€ 9,291)$. We estimate the cost for ten 1 GbE connections at $€ 15,500$ and for one $1 \mathrm{GbE}$ connection at $€ 1,550$. The cost for a $10 \mathrm{GbE}$ connection is estimated as four times the cost of a $1 \mathrm{GbE}$ connection $(€ 6,200)$. We estimate the yearly cost for connecting Ghent with Brussels $(75 \mathrm{~km})$ at $€ 37,500$ per dark fiber.

\section{E. Results}

Convergence: By reducing the number of networks, both network capital expenditures (CapEx) and operational expenditures (OpEx) may decrease. In the analysis, we focus on network CapEx. Figure 3 summarizes the obtained results per scenario. These show that today it is $20 \%$ more cost-efficient to choose for a converged network based on IP technology (scenario 2$)^{3}$.

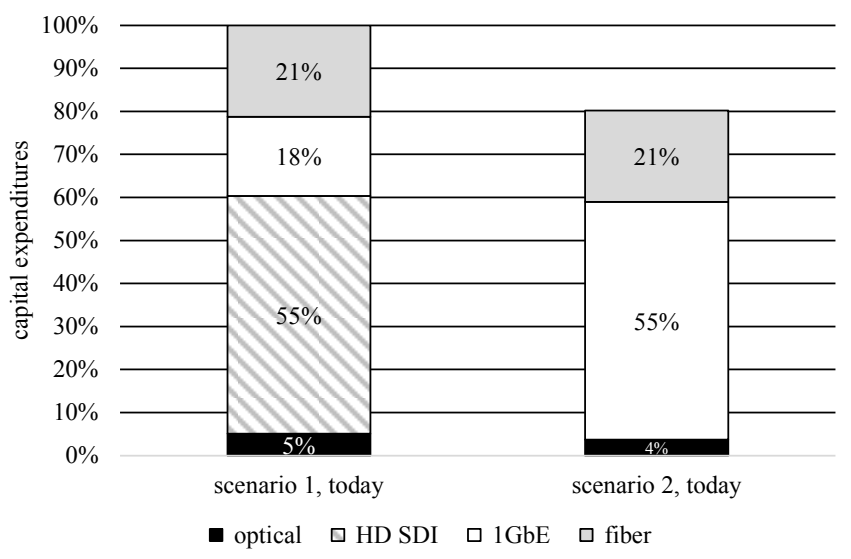

Fig. 3. Comparison between scenario 1 and 2 for todays traffic sources

Decoupling: As technology evolves, both end devices and the network infrastructure needs to be upgraded to follow the latest trends (e.g. migration from HD to 4K). Certain networks however are not format-agnostic. For example, an upgrade from HD SDI to $4 \mathrm{~K}$ SDI requires new end devices, new interfaces, new cabling and new routers. An IP network on the

\footnotetext{
${ }^{3}$ we assume that it is possible to send a HD stream over a $1 \mathrm{GbE}$ connection
}

other hand may have, to some degree, reusable components. For example, it may be adequate to upgrade the router line card from $1 \mathrm{GbE}$ ports to $10 \mathrm{GbE}$ ports to instead of buying a completely new router.If we take these aspects into account, the converged scenario shows a cost benefit of $27 \%$ compared to the unconverged scenario (4).

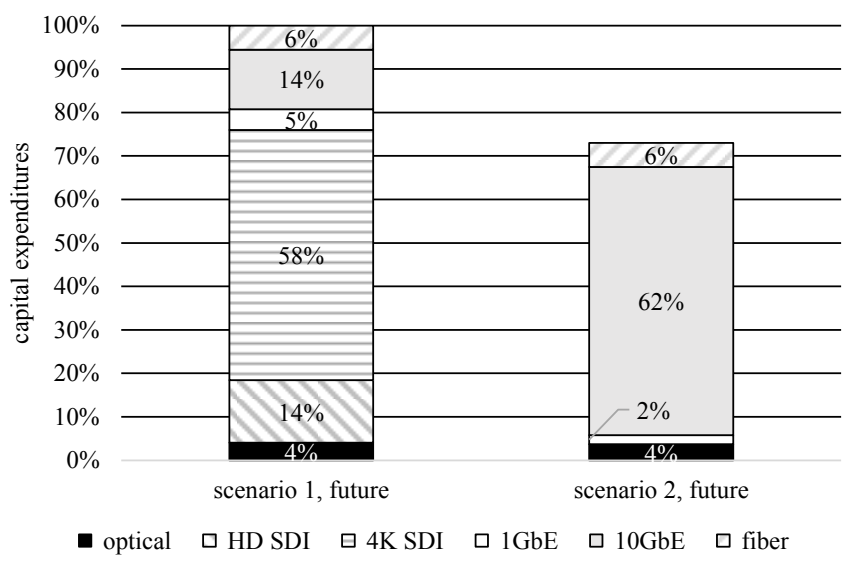

Fig. 4. Comparison between scenario 1 and 2 for future traffic sources

\section{F. Refine}

Before concluding the techno-economic analysis we should point to a number of factors that likely could have an impact on our analysis. These include uncertainties in the input data and factors that are not taken into account because they were out of scope:

1) the analysis uses cost input data that are based on official price lists. In practice, large discounts are applied to these prices (up to 50\%) and on top of that quantity discounts may be applied. Prices also vary largely between vendors and depend on the expected reliability. This will clearly have an impact on the obtained results but should not change the relative cost benefit of scenario 2 compared to scenario 1

2) the analysis uses technologies which are not yet available on the market or that are rather new. An example is the price of a $4 \mathrm{~K}$ SDI line card. Today, these are not yet available. As such, prices were estimated based on historical data and discussion with industry experts. Similarly the price of $10 \mathrm{GbE}$ connections will likely evolve in the future (become cheaper) as the need for these increases.

3) the analysis takes two types of networks into account (an SDI network and a QoS IP network), in reality more parallel networks exist (e.g. a traditional IP network). We expect this to contribute further to the advantage of the converged scenario.

4) the analysis focuses on the media distribution and contribution network (wide area network). Even though this is the core network, connectivity has to be provided to the end points (e.g. cameras, microphones, etc.). The 
cost of that (network) equipment is not included. We expect this to contribute further to the advantage of the converged scenario.

\section{G. Market barriers}

Even though the results of this paper clearly indicate the cost benefits of converging to one IP network, there are still a number of non-technical barriers to be overcome before the media sector is fully ready for this evolution. As a final section to this paper, we list here a few:

1) Ability of a business to lease a dark fiber connection. More often than not, a dark fiber connection cannot be leased by the involved parties because no fiber is available or the fiber operators only offer is an MPLS connection. As the available bandwidth in such cases is constrained, the effective management of that connection is very much needed.

2) Pricing of dark fiber or dedicated high-bandwidth connection. If a dark fiber can be leased, the pricing consists of a one-time connection cost and a recurring usage fee per $\mathrm{km}$ of fiber. The recurring usage fee varies widely between countries, providers and even between customers.

3) Willingness to migrate from SDI-based media production to IP production. Up until now, professional media networks rely on Serial Digital Interface (SDI) cabling, routers and switches. Due to the fast pace of innovation the costs associated with upgrading the infrastructure is increasing. Therefore, an agnostic infrastructure, such as an IP network, that is able to support future resolutions and services could have its benefits. By encapsulating video over IP, different signals can be aggregated and transported over a single link instead of using a single link per SDI stream. As such, the utilization of transport links can be improved and hardware costs can be reduced by the economies of scale involved with standardized versus SDI-specific equipment.

4) Change in working habits of (post)production employees. The new collaboration models proposed above may well optimize the use of dedicated equipment and reduce travel costs, they also request a shift in behavior from the employees. Nowadays, everyone has its own, dedicated editing cell, frequently on the same location as where the program is actually recorded. This allows for a direct interaction between the production and editing team. In the future, remote processes will remove the need for on-location editing, but should of course allow for other types of interactions as to not negatively influence the creative process.

\section{CONCLUSION}

This paper discusses the media sector and in particular the way transport of media files is done today. We presented an alternative approach in which the media production system is converged onto an IP network with stringent performance guarantees. We introduced the technology and conducted a techno-economic study showing a cost benefit of $27 \%$ compared to current solutions.

\section{ACKNOWLEDGMENT}

This research was carried out as part of the iMinds MECaNO project. This project is co-funded by iMinds (Interdisciplinary Institute for Broadband Technology) a research institute founded by the Flemish Government. Companies and organizations involved in the project are SDNsquare, Vlaamse Radio en Televisieomroeporganisatie (VRT), Videohouse, Limecraft, Alcatel-Lucent, Ghent University and University of Antwerp with project support of IWT.

\section{REFERENCES}

[1] "MECaNO (media contribution network optimization)." [Online]. Available: https://www.iminds.be/en/projects/mecano

[2] H. Zettl, Television production handbook. Cengage Learning, 2011.

[3] P. Trimintzios, T. Baugé, G. Pavlou, P. Flegkas, and R. Egan, "Quality of service provisioning through traffic engineering with applicability to ipbased production networks," Computer Communications, vol. 26, no. 8, pp. 845-860, 2003

[4] N. Harris, "Sohonet: a case study in real-world media networking," in ATM in Professional and Consumer Applications (Digest No: 1997/113), IEEE Colloquium on. IET, 1997, pp. 5-1.

[5] T. Kojima, J. J. Stone, J.-R. Chen, and P. N. Gardiner, "A practical approach to ip live production," Motion Imaging Journal, SMPTE, vol. 124, no. 2, pp. 29-40, 2015.

[6] S. Verbrugge, K. Casier, J. Van Ooteghem, and B. Lannoo, "Practical steps in techno-economic evaluation of network deployment planning part 1: methodology overview," in 13th international telecommunications network strategy and planning symposium (networks 2008). IEEE, 2008.

[7] "WeTransfer." [Online]. Available: https://www.wetransfer.com/ 